E-MAIL CONTRIBUTIONS

\title{
Stress-forecasting: an apparently viable third strategy
}

\section{STUART CRAMPIN}

\begin{abstract}
All discussions so far have referred (perhaps not surprisingly) to the properties of earthquakes, their times, locations, nucleation mechanisms, physics of the source, possible precursors, etc. I think this will lead nowhere. Earthquakes are extraordinarily varied and impossible to average. Perhaps the only feature of earthquakes that can be relied on is that they release a large amount of stress which, because rock is weak, has necessarily accumulated over a large volume of rock. If this build up of stress can be monitored then the time and magnitude of the earthquake when fracture criticality is reached can be subject to 'stress-forecast'. I suggest that we already know how to do this. The effects have been seen with hindsight for eight earthquakes worldwide, and the time and magnitude of an $\mathrm{M}=5$ earthquake has been successfully stress-forecasted.
\end{abstract}

Let me try to introduce a little realism into the debate. Earthquakes are complex. They vary: with magnitude and direction of stress-field; shape of the fault planes; orientation of fault plane with respect to stress field; presence or absence of fluids; nature of fluids; fluid-pressure; asperities on fault plane; debris on fault plane; presence or absence of fault gouge; presence or absence of water channels, pressure seals; height of water table; temperature; state of Earth tides; state of ocean tides; air pressure; local geology; other earthquakes; and so on and so on. Each of these phenomena could in certain circumstances have major effects on time, place, and magnitude of impending earthquakes. Consequently, no two earthquakes are identical (although seismic records being dominated by the ray path may be very similar).

To understand, model, and accurately predict the behaviour of such a source requires intimate knowledge of every grain of the fault gouge and every microcrack in the rockmass. This might be possible in theory but in practice is totally unknowable by tens of orders of magnitude (and similarly beyond the capacity of any existing or foreseeable computer to model or manipulate again by tens of orders of magnitude). Earthquake prediction is not just a difficult subject where more knowledge or funding is required, it is out of our reach by astronomical-sized factors.

This is the reason, why techniques which depend on any feature of the source, or any source-induced precursors, understanding nucleation processes, etc., are not likely to succeed. There is just far to much heterogeneity by once more tens of orders of magnitude. It is pretty clear by now that there is no magic formula, waiting to be discovered as some of the discussions seem to imply. So Bob Geller's first entry in this debate is correct on the basis of looking at the earthquake source, prediction of the time, place, and magnitude is practically impossible. There is just far too much possible variety.

Consequently, it is hardly surprising that the classical earthquake prediction of time, magnitude and place of future earthquakes within narrow limits seems impossible. The claims of success listed by Max Wyss seem extremely shaky. One may wish for something to turn up, as some in this debate have done, but I suggest that it is clear from any contemplation of the enormous complexity and variability of the earthquake source that such hopes are futile and not worth wasting time or spending money on.

Can we do anything? I believe we can, but not by examining the source. Rock is weak to shear stress, which means that the stress released by earthquakes 
has to accumulate over enormous volumes of rock. Perhaps hundreds of millions of cubic kilometres before an $\mathrm{M}=8$ earthquake. There is mounting direct and indirect evidence $\frac{1-4}{4}$ that changes in seismic shear-wave splitting (seismic bi-refringence) can monitor the necessary build up of stress almost anywhere in the vast stressed rockmass before the earthquake can occur.

Most rocks in the crust contain stress-aligned fluid-saturated grain-boundary cracks and pores ${ }^{-1}$. These are the most compliant elements of the rockmass and their geometry is modified by the build up of stress $2,3,5$. Variations in seismic shear-wave splitting reflect changes of crack geometry, and hence can monitor the build-up of stress before earthquakes ${ }^{2}$ and the release of stress at the time of (or in one case shortly before) the earthquake. Such changes have been identified with hindsight before three earthquakes in USA, one in China ${ }^{3,5}$, and now routinely before four earthquakes in SW Iceland 6 (Please see these references for further details of these studies).

The interpretation of these changes in shear-wave splitting is that stress builds up until the rock reaches fracture criticality when the cracking is so extensive that there are through-going fractures (at the percolation threshold) and the earthquake occurs 2,6 . The rate of increase of stress can be estimated by the changes in shear-wave splitting, and the level of fracture criticality from previous earthquakes. When the increasing stress reaches fracture criticality the earthquake occurs. Magnitude can be estimated from the inverse of the rate of stress increase ${ }^{-6}$ : for a given rate of stress input, if stress accumulates over a small volume the rate is faster but the final earthquake smaller, whereas if stress accumulates over a larger volume the rate is slower but the earthquake larger.

As of $17^{\text {th }}$ March, 1999 one earthquake has been successfully stress forecast in real-time giving the time and magnitude of a $M=5$ earthquake in SW Iceland $\underline{6}$. Non-specific stress-forecasts were issued to the Icelandic National Civil Defence Committee on the $27^{\text {th }}$ and $29^{\text {th }}$ October, 1998. The final timemagnitude window (a window is necessary because of uncertainties in estimates) on $10^{\text {th }}$ November, 1998 , was a $\mathrm{M}>=5$ soon or, if stress continued to increase, a $M>=6$ before the end of February 1999 . Three days later (13 ${ }^{\text {th }}$ November, 1999), there was a $M=5$ earthquake within $2 \mathrm{~km}$ of the centre of the three stations where changes in shear-wave splitting were observed. We claim this is a successful real-time stress-forecast, as anticipated from the behaviour noted with hindsight elsewhere. Shear-wave splitting does not indicate potential earthquake locations, but analysis of local seismicity by Ragnar Stef? nsson correctly predicted the small fault on which the stress-forecast earthquake occurred. It appears that monitoring the build up of stress before earthquakes can forecast the time and magnitude of impending earthquakes.

Three comments about stress-forecasting:

1. Stress-forecasting seems to give reasonable estimates of time and magnitude but gives little or no information about location, where perhaps Bob Geller's stochasticism takes over. However, as Chris Scholz says, "earthquake prediction is always local". If it is known that a large earthquake is going to occur (that is when there has been a stress-forecast), local information may be able to indicate the fault that will break, as happened in Iceland.

2. Stress-forecasting was possible in SW Iceland only because of the unique seismicity of the onshore transform-zone of the Mid-Atlantic Ridge where nearly-continuous swarm activity provided sufficient shear-waves to illuminate the rockmass. Routine stress-forecasting elsewhere, without such swarm activity, would require controlledsource seismology.

3. The phenomena we are observing are not precursors. Apart from the decrease in stress at the time of the earthquake, the effects are independent of the earthquake source parameters. Shear-wave splitting monitors a more fundamental process, the effects of the build up of 
stress on the rockmass, which allows the estimation of the rate of increase and the time when fracture criticality is reached.

For reasons not fully understood, but probably to do with the underlying critical nature of the non-linear fluid-rock interactions ${ }^{4.7}$ the effect of the stressed fluid-saturated microcracks on shear-waves is remarkably stable ${ }^{1-3,5}$.

We see exactly the same behaviour before the 1996 Vatnaj kull eruption in Iceland as we see before earthquakes. About 1 cubic kilometre of magma was injected into the crust over a five month period. The major difference from an earthquake being that the stress was not abruptly released by the eruption, as it would have been by an earthquake, following the eruption the stress relaxed over a period of several years as it was accommodated by a spreading cycle of the Mid-Atlantic Ridge.

I suggest that monitoring the build up of stress is a third strategy for predicting earthquakes beyond the two - detecting precursors, and detailed modelling of earthquake physics - suggested by Dave Jackson. Like many features of shear-wave splitting, it appears to be comparatively stable, appears to have considerable accuracy in forecasting time and magnitude.

\section{Stuart Crampin}

Centre for Reservoir Geoscience, Department of Geology \& Geophysics, University of Edinburgh, Grant Institute, West Mains Road, Edinburgh EH9 3JW, SCOTLAND

email:scrampin@ed.ac.uk.

\section{References}

1. Crampin, S. The fracture criticality of crustal rocks. Geophys. J. Int. 118, 428-438 (1994).

2. Crampin, S. \& Zatsepin, S. V. Modelling the compliance of crustal rock, II - response to temporal changes before earthquakes. Geophys. J. Int. 129, 495-50 (1997).

3. Crampin, S. Calculable fluid-rock interactions. J. Geol. Soc. (in the press) (1999).

4. Crampin, S. Shear-wave splitting in a critical crust: the next step. Rev. Inst. Fran. Pet. 53, 749-763 (1998).

5. Crampin, S. Stress-forecasting: a viable alternative to earthquake prediction in a dynamic Earth. Trans. R. Soc. Edin., Earth Sci. 89, 121-133 (1998).

6. Crampin, S., Volti, T. \& Stefansson, R. A successfully stress-forecast earthquake. Geophys. J. Int. (in the press) (1999).

7. Crampin, S. Going APE: II - The physical manifestation of selforganized criticality. 67th Ann. Int. Mtg. SEG, Dallas, Expanded Abstracts, 1, 956-959 (1997).

Contributions to this debate from readers are encouraged, by e-mail only, please, to debates@nature.com.

The rules are simple: contributions should be short and to the point. The moderator reserves right to select contributions to be posted on the site, and to normal editing for style, sense, length and, of course, good taste. 